\title{
Shopping Center Tracking and Recommendation Systems
}

\author{
Ricardo Anacleto, Nuno Luz, Ana Almeida, Lino Figueiredo and Paulo Novais
}

\begin{abstract}
Shopping centers present a rich and heterogeneous environment, where IT systems can be implemented in order to support the needs of its actors. However, due to the environment complexity, several feasibility issues emerge when designing both the logical and physical architecture of such systems. Additionally, the system must be able to cope with the individual needs of each actor, and provide services that are easily adopted by them, taking into account several sociological and economical aspects. In this sense, we present an overview of current support systems for shopping center environments. From this overview, a high-level model of the domain (involving actors and services) is described along with challenges and possible features in the context of current Semantic Web, mobile device and sensor technologies.
\end{abstract}

Key words: Mobile, Shopping Center, Tracking, Recommendation, Marketing

\section{Introduction}

Although some approaches to shopping center tracking and/or recommendation systems already exist, they are still difficult to implement in real scenarios due to the several challenges such an environment presents, and are still far from being recognized for their applicability and possible features that can support all actors and entities involved in the shopping center context.

Such a system must address the needs of every actor (e.g., visitors, sellers, administrators and security) involved in the shopping center environment. In this sense,

Ricardo Anacleto, Nuno Luz, Ana Almeida and Lino Figueiredo

GECAD - Knowledge Engineering and Decision Support, R. Dr. António Bernardino de Almeida, 431. 4200-072 Porto, Portugal, e-mail: \{rmao, nmal, amn, lbf $\}$ isep.ipp.pt

Paulo Novais

Universidade do Minho, Campus of Gualtar. 4710-057 Braga, Portugal, e-mail: pjon@di.uminho.pt 
different features must be provided according to each type of actor and its specific personal profile. For example, it might be intended that every seller builds its own interactive space in a virtual shopping center area, thus allowing the sellers' presence in entertainment, marketing and publicity events.

In this paper, we present the current state of the art in shopping center tracking and recommendation systems. We follow with the challenges imposed by such systems according to current technologies and approaches in tracking and recommendation systems. Next, we present several features that can be useful in different actor type perspectives. Finally, some conclusions and future work are presented.

\section{State of the Art}

With the growing popularity and evolution of mobile devices and sensors, several application scenarios previously left in the dark due to feasibility problems are being explored. One of these scenarios where mobile devices can be useful when building and designing support systems is shopping centers.

In [2], an indoor wireless system for personalized shopping assistance composed by a central service and mobile devices is proposed. Specific mobile devices were developed to guide the user through the store, providing details about products of interest via the built-in device screen. Sale items are highlighted and a comparative price analysis is presented, reminding the user of unavailable items that he has been looking for in the past that are now available. Also, the user is able to find the current price of an item by scanning its bar code with a built-in bar code reader. At the same time, the devices media player plays music according to the user profile, built from previous interactions with items.

The system integrates wireless, video, speech and real-time data access technologies to personalize the experience provided to a customer, based on individual needs and a shopping profile updated over time.

The centralized server maintains the product database, store database, and customer profiles, providing audiovisual responses to inquiries from tens to hundreds of customers in real-time over a wireless network.

In an experimental scenario, upon establishing communications with the mobile device, the central service queries the customer database and sees that the user recently purchased a VCR (VideoCassette Recorder). In greeting the user, it announces I thought you might be interested to know that BASF video tapes are on sale today. Also, the system contains story telling features to keep kids entertained and occupied.

However, the system is very expensive since it requires specific mobile devices. Because almost every person has a mobile phone, which is able to cover most of the hardware needs in such a system, selling specific mobile devices might not be a feasible solution. Additionally, RFID (Radio-Frequency IDentification) tags are distributed all over the shopping center, increasing costs. 
In [8], a customized one-to-one recommendation system inside a virtual shopping center, considering server, product, and facility at the same time, is proposed. To cope with these considerations, the system detects purchase intention patterns by clustering purchase history of target customers. It can also understand the characteristics of each customer's purchase. After grasping some noticeable purchase patterns, it determines a recommendation according to the customer's location inside the virtual shopping. By means of recommending items according to the path of each customer (location-awareness), an improvement in sales and profit of a retail company is expected. Also, the system stores huge amounts of information that can be used personalized and directed marketing campaigns.

In [17], the shopping center system tries to explore how to assist the user in achieving his goal of the best possible buying decision within a given limited time. Since navigation assistance sometimes becomes necessary to help the user find what he wants, the system tracks the location of the user through RFID tags. Displays are distributed throughout the shopping center in order to present multimedia content and navigational instructions to the user. However, using the displays as a shared medium imposes scalability problems.

The system is described like this: imagine a user, who has just checked in at a large airport and wants to buy a digital camera at the duty-free zone before the boarding of the plane. The first goal is to find electronics store which offers a good selection of digital cameras. The user activates his PDA (Personal Digital Assistant) to explore the environment, picks a store and requests navigational aid.

Another approach is a context-aware content-provision service [18], in which the content is assumed to be commercial advertisements in a shopping center. The users can enjoy many types of the content-provision services based on their own profiles. The system detects context, determines suitable content according to context, and provides the content to the user through a mobile device. Here, the context includes present attribute data of the user, and content from stores. RFID tags are used to locate the users.

Sae-Ueng et al. propose a consumer-friendly shopping assistance service based on personal behavior data in ubiquitous shopping spaces [14]. The system tries to study the personal behavior using RFID and camera sensors in the ubiquitous environment to build an user profile. Later on, the consumer-friendly information services present, through display monitors, new available products.

Apriori presents a concept that enables customers to access and share product recommendations using their mobile phones [12]. Based on a review of current product recommendation mechanisms, it leverages the potential of auto-ID-enabled mobile phones (barcode/RFID) to receive and submit product ratings. Basically, it acts as a collaborative recommendation system, storing customer ratings to classify the products in the shopping center.

A good shopping recommender system can boost sales in a retailer store. To provide accurate recommendation, the recommender needs to accurately predict a customer's preference, an ability difficult to acquire. Chun-Nan et al. [6] reported their experience in data mining skewed and sparse transaction data sets to predict individual customers shopping preferences for two large retailer stores. From previous 
experiments, they concluded that collaborative ltering methods such as GroupLens [13] and association-rule based methods such as the IBM (International Business Machines) method [9], can generally be applied to this problem but rarely produce satisfying results. In this sense, they propose HyPAM (Hybrid Poisson Aspect Modeling), a probabilistic graphical model to address the issues of data skewness and sparsity.

B-MAD system (Bluetooth Mobile ADvertising) [1] delivers location-aware mobile advertisements to mobile phones using Bluetooth positioning and Wireless Application Protocol (WAP) push, with an accuracy of 50 to 100 meters.

Nine Bluetooth sensors (Nokia 3650 phones running the Bluetooth sensor software) were placed in the display windows of eight retail stores around the Rotuaari area, which includes pedestrian streets at the center of the city of Oulu in Northern Finland. Experimental results showed that the system provides a viable solution for realizing mobile advertising, but not with a poor accuracy of 50 to 100 meters. Also, advertisements weren't profiled for each user.

In [10], a project concerned with designing and implementing a Internet shopping center by using a virtual reality-driven avatar and web decision support system is described. The virtual reality technique guarantees a sense of reality for the customers' and facilitates the complex process of decision making in shopping.

SHOMAS Multiagent System [3] provides guidance, planning, suggestions of products and advertising promotions for shopping center users. Also it can track people arround the mall to use, for example, when a child is lost.

The multiagent architecture incorporates reactive and deliberative agents that take decisions automatically in execution time. The architecture of SHOMAS (see fig. 1) is composed by "lightweight" agents that are downloaded to the user wireless mobile device (e.g. PDA), which interact with a central agent (using a case-based planning system). Also, every store in the shopping mall incorporates one agent to calculate the optimal promotions (those of greater sales success) and services at a given moment by considering retail data and user profiles.

To provide security and to optimize users time in the mall, user location must be known, so this system uses RFID technology to get users location. Each user have a RFID tag mounted on braceletsworn on thewrist or ankle to identify the user. The door readers sensors are installed in strategic areas within themall. Each reader sends a pulse of radio energy to the tags and listen for the tags response.

To advertise the system uses past experiences, user profile and maximum amount of money to find the best advertises with the concepts Believe, Desire and Intention. Each user must fill in a inquiry, then user prole is updated from retail data and periodic questionnaires.

The system described in this paper was tested in a Shopping Mall in the city of Salamanca, Spain during 2005 and 2006. The overall user satisfaction was about $65 \%$ during the 16 months of the experiment. Other metrics were used to classify the system, for example, comparing the sales rates with past years, which demonstrates that the system helped sales growing but another reasons can explain that conclusion (like economical crises, etc). 


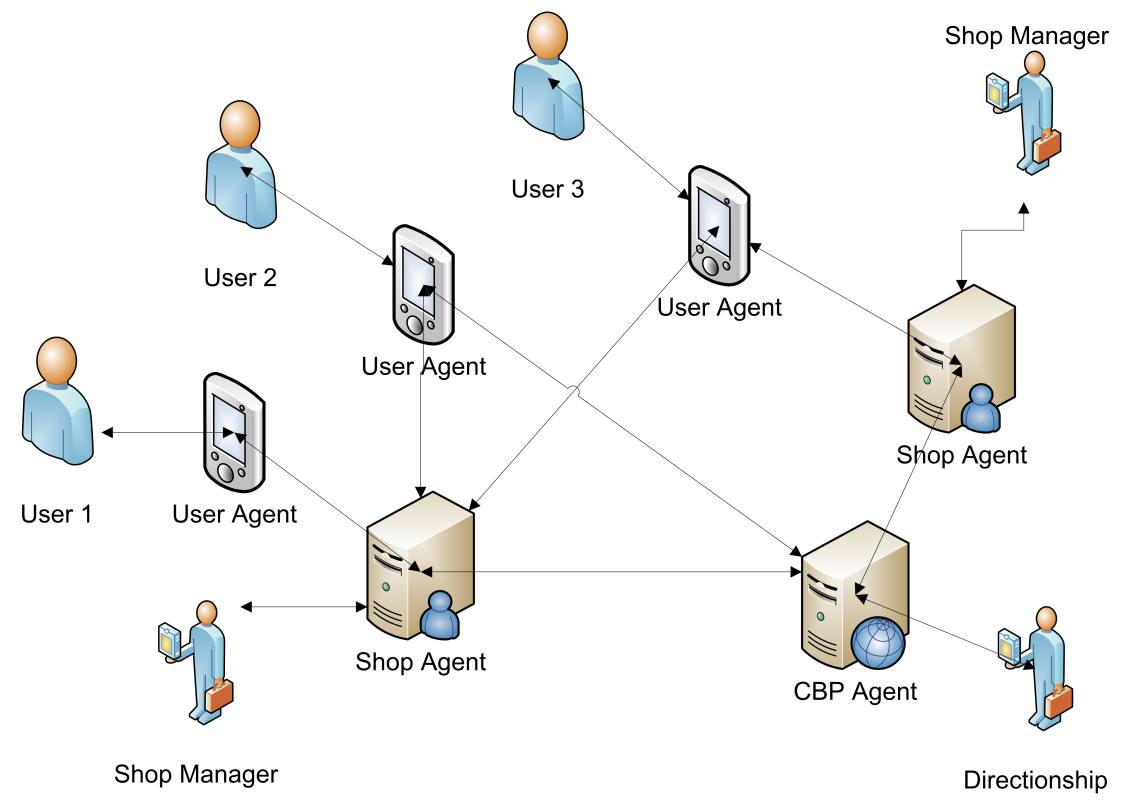

Fig. 1 High level taxonomy of actors and services in a shopping center environment.

\section{Challenges in Technologies and Architectures}

A shopping center environment involves a wide variety of actors with different purposes and needs. In that sense, several services and applications can be devised and oriented to a specific type of actor. Both actors and services can be classified according to a simple taxonomy as the one presented in fig. 2 .

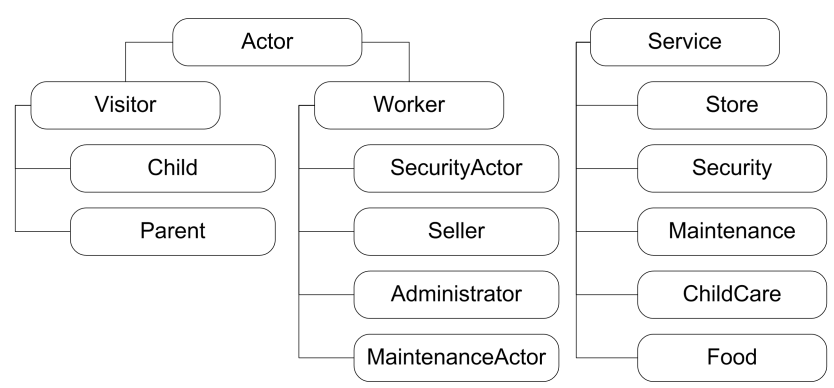

Fig. 2 High level taxonomy of actors and services in a shopping center environment.

Notice that although the taxonomy provides a classification of actors and services, it does not model how these actors interact with the several services provided by the system. Such a model can be achieved through an ontology representation of 
the knowledge domain. This way, and with the flexibility of a service-oriented architecture, the semantics of the interactions between services and actors is explicitly modeled, so that they can be easily added and removed from the system.

Due to the extensibility and inherent semantics of ontologies, they can be used to ensure that all interconnected services understand each other, to perform reasoning over the shopping center environment data, and by stores to extend the high level ontology and define an internal classification of actors and products. Additionally, reasoning mechanisms can be set over the existing knowledge base in order to infer new implicit facts. In this specific case, non-monotonic reasoning offers a more natural way of inferring knowledge [4].

Each service can provide important information about the activity of an (usually business) entity, such as products, events, maps of the entity space information and all kinds of data belonging to, for example, the store context.

However, such an heterogeneous infrastructure imposes challenges that cannot be fully covered with current technologies. Although the Semantic Web has boosted the popularity of ontologies and related languages and tools, triple store management systems still lack in performance when compared to well established relational database management systems. Also, non-monotonic reasoning has only been recently explored in a handful of technologies such as OWL [7], RIF-PRD (Rule Interchange Format Production Rules Dialect) [11] and Pellet (through the K operator) [16], and the expressiveness of the employed ontology language must be carefully chosen, so reasoning processes are able to infer new knowledge deterministically.

Securing mobile device communications can be difficult due to wireless communications. To secure the system, all communications must be encrypted so no sensitive data leaks to third party users. Authentication and authority is very important, so every actor and service in the system must be correctly identified and have its own profile.

In order to reason and deliver personalized services to the shopping actors, the system must be able to capture the context of each actor inside the shopping center, which might involve several dimensions such as location, user interests, and current beliefs, desires and intentions (BDI).

Additional issues emerge when dealing with the physical infrastructure, since several sensors must be deployed for the system to capture context. Mobile devices also present some challenges regarding display size, battery life and network communications, thus forming a substantial amount of concerns that must be tackled when designing and implementing the system.

Since localization technologies like GPS (Global Positioning System) don't work inside buildings and certain locations difficult to reach. To overcome this limitations there are several indoor location systems based on infra-red, ultrasound, narrowband radio, Wi-Fi signal strength, UWB (Ultra-WideBand), vision, and many others [5]. However, few can be easily deployed over large buildings, due to the necessity for a structured environment. This can be a possible solution when GPS isn't available, but it is very expensive to implement and it has a large error rate, since the environment is constantly changing. 
To remove this limitation, a system that allows indoor location becomes necessary, such as an Inertial Navigation System (INS) [15]. This type of systems consist of Micro Electrical Mechanical System (MEMS) devices which communicate with the central module using a wireless network (Bluetooth). These devices obtain information of the movements of an individual independently of the infrastructure of the building. All this sensory set requires the implementation of a sensor fusion, so algorithms are implemented to interpret the information from sensors and thereby determine the position of the individual. The information collected, in addition to the speed and direction of motion, must also be able to determine the step width and position of the individual (sitting, lying or standing). To get this information several sensors are spread in the body, on the foots are pressure and accelerometers sensors, and on the hip there are gyroscope and compass sensors. They must be imperceptible to the user, to not bother the visitor when he is shopping.

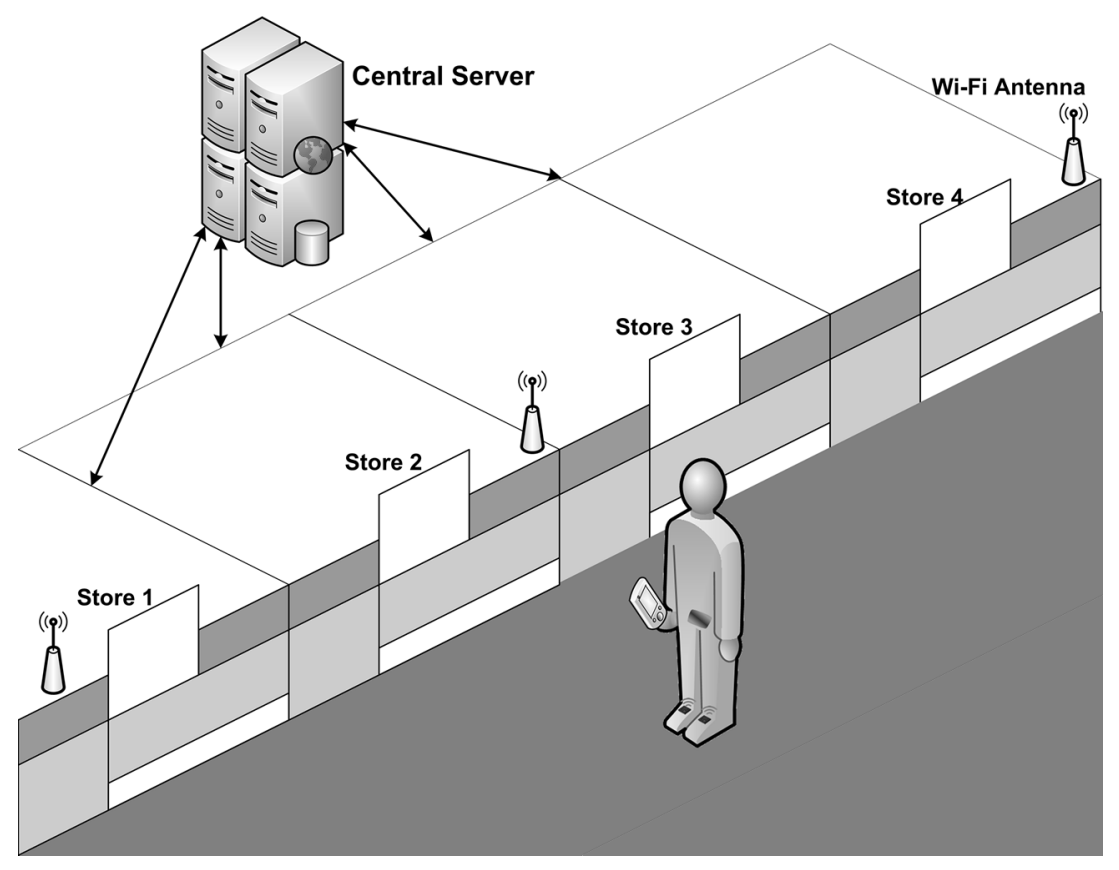

Fig. 3 System Architecture Example

As can be seen in fig. 3 every store, present in the shopping center, is connected to a central server (that is a cluster of servers: database server, web server, etc.), where each one put their promotions, marketing campaigns, products price, etc. In this server the personal profile of each user is stored. The personal profile is obtained according to purchase items history. When the shopping visitor enters in the shopping center, he gets its PDA and connects to the Wi-Fi network of the building, then opens the shopping mobile application. 
This application runs in background waiting for messages that come from central server. The server sends notifications to visitors PDA, that corresponds to his profile, for example, if he likes reading and the shopping center book store is giving a discount of $20 \%$ on the next two hours, a notification is sent to him.

\section{Applicability}

For each actor involved in the system, useful different services can be provided (see fig. 4). These include object tracking (e.g., cars, products), location guidance (locate available services), children monitoring, disturbance detection, targeted personalized announcements (context and location-aware), and specific marketingcampaigns involving games and events.

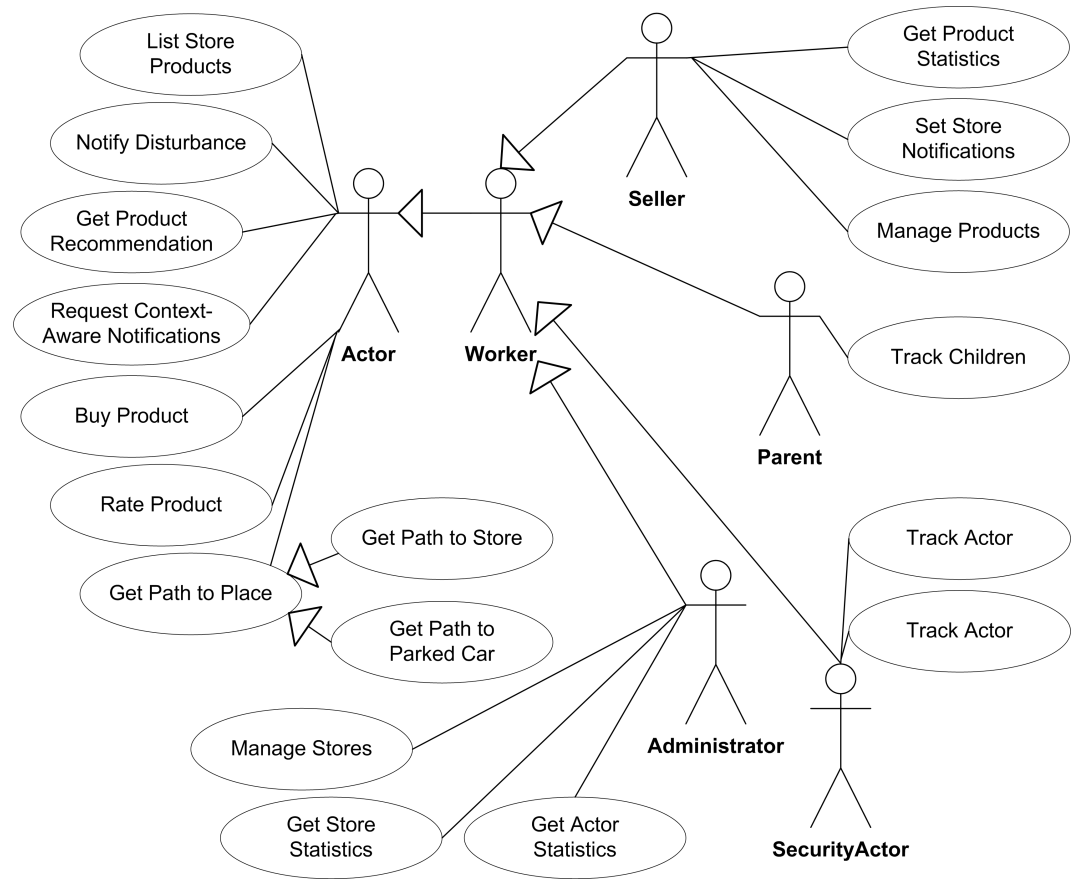

Fig. 4 Some use cases for some of the actors in the taxonomy.

From gathered data, a visitor profile can be built and used in a recommendation system in order to recommend new and interesting products to the visitor. Besides, collaborative filtering techniques can be used, as long as the system interacts with widespread online social networks to collect friend information and provide recommendations. 
Tracking the actor from the moment he enters the shopping center is mandatory in order to provide location-aware services. Such services include parking location guidance (for those that forget where the car is parked), location-aware announcements and notifications about products, immediate disturbance detection, children monitoring and guidance through the entire shopping center. However, this might be seen as a privacy breach by some of the shopping center visitors.

Additional services include the existence of a virtual space for each seller, allowing visitors to search through available products. Cinemas can also benefit from such a system, since tickets can be obtained and paid for through the mobile device. Treasure hunting events can be used as a marketing maneuver and to entertain at the same time. Any visitor can participate on the event and search for a treasure, while overcoming obstacles. These obstacles might try to capture the visitors' attention into certain stores and products. The winner can be given credits to buy items at the shopping center.

\section{Conclusions}

Implementing IT support systems in shopping center environments presents many challenges due to the necessary infrastructure and the richness and heterogeneity of the domain. However, current technologies, such as those emerging from the Semantic Web and tracking systems, are evolving in a direction where these challenges can be tackled.

The different business and maintenance areas involved in the shopping center environment offers possibilities for the implementation of many features. These can be specifically oriented to marketing, administrative, advertisement and entertainment purposes.

In future work, we intend to explore, in particular, the feasibility of an ontologybased architecture, where the presented actor taxonomy is extended, and complemented with a service ontology providing flexibility and enriched semantics for reasoning engines. This involves both the use and specification of domain specific ontologies and rules. The use of ontologies also leverages the possibility of integrating more than one shopping center system. In this case, if different ontologies representing the same (or overlapped) domains are used, an ontology mapping process must be applied.

Tracking accuracy is essential in such a system. In this sense, future work also involves research in tracking using MEMS, with the aim of providing location-aware information in unstructured environments where GPS signal doesn't reach.

Additionally, the feasibility of such a solution must be evaluated according the different dimensions of the problem, which involves several sociological, economical and technological aspects. 


\section{References}

1. L. Aalto, N. Gthlin, J. Korhonen, and T. Ojala. Bluetooth and WAP push based location-aware mobile advertising system. In Proceedings of the 2nd international conference on Mobile systems, applications, and services, page 4958, 2004.

2. A. Asthana, M. Cravatts, and P. Krzyzanowski. An indoor wireless system for personalized shopping assistance. In Mobile Computing Systems and Applications, 1994. Proceedings., Workshop on, page 6974, 2002.

3. J. Bajo, J. Corchado, Y. Paz, J De Paz, S. Rodrguez, Q. Martn, and A. Abraham. SHOMAS: Intelligent guidance and suggestions in shopping centres. In Applied Soft Computing Volume 9, Issue 2, March 2009, page 851862, 2009.

4. K. Frankish. Non-monotonic inference. The Encyclopedia of Language and Linguistics, (2), 2005.

5. J. Hightower and G. Borriello. Location systems for ubiquitous computing. IEEE Computer, 34(8):5766, 2002.

6. C. N Hsu, H. H Chung, and H. S Huang. Mining skewed and sparse transaction data for personalized shopping recommendation. Machine Learning, 57(1):3559, 2004.

7. Y. Katz and B. Parsia. Towards a nonmonotonic extension to OWL. In Proceedings of Workshop on OWL Experiences and Directions, Galway, Ireland, 2005.

8. T. Kim and S. C Park. One-to-one customized brand recommendation in virtual shopping mall. In Management of Innovation and Technology, 2000. ICMIT 2000. Proceedings of the 2000 IEEE International Conference on, volume 2, page 659663, 2002.

9. R. D Lawrence, G. S Almasi, V. Kotlyar, M. S Viveros, and S. S. Duri. Personalization of supermarket product recommendations. Data Mining and Knowledge Discovery, 5(1):1132, 2001.

10. K. Chang Lee and N. Chung. A web DSS approach to building an intelligent internet shopping mall by integrating virtual reality and avatar. Expert systems with applications, 28(2):333346, 2005.

11. A. Polleres. Semantic web technologies: From theory to standards. In 21 st National Conference on Artificial Intelligence and Cognitive Science, NUI Galway, 2010.

12. F. Von Reischach, D. Guinard, F. Michahelles, and E. Fleisch. A mobile product recommendation system interacting with tagged products. In Pervasive Computing and Communications, 2009. PerCom 2009. IEEE International Conference on, page 16, 2009.

13. P. Resnick, N. Iacovou, M. Suchak, P. Bergstrom, and J. Riedl. GroupLens: an open architecture for collaborative filtering of netnews. In Proceedings of the 1994 ACM conference on Computer supported cooperative work, page 175186, 1994.

14. S. Sae-Ueng, S. Pinyapong, A. Ogino, and T. Kato. Consumer-Friendly shopping assistance by personal behavior log analysis on ubiquitous shop space. In Asia-Pacific Service Computing Conference, The 2nd IEEE, page 496503, 2007.

15. P. G Savage. Strapdown inertial navigation integration algorithm design part 1: Attitude algorithms. Journal of guidance control and dynamics, 21:1928, 1998.

16. E. Sirin, B. Parsia, B. C Grau, A. Kalyanpur, and Y. Katz. Pellet: A practical owl-dl reasoner. Web Semantics: science, services and agents on the World Wide Web, 5(2):5153, 2007.

17. C. Stahl, J. Baus, B. Brandherm, M. Schmitz, and T. Schwartz. Navigational and shopping assistance on the basis of user interactions in intelligent environments. In The IEEE International Workshop on Intelligent Environments, page 182191, 2005.

18. Y. Yokohata, Y. Yamato, M. Takemoto, E. Tanaka, and K. Nishiki. Context-aware contentprovision service for shopping malls based on ubiquitous service-oriented network framework and authentication and access control agent framework. In Consumer Communications and Networking Conference, 2006. CCNC 2006. 3rd IEEE, volume 2, page 13301331, 2006. 